Pure and Applied Mathematics Quarterly

Volume 1, Number 4

(Special Issue: In Memory of

Armand Borel, Part 3 of 3 )

$791-815,2005$

\title{
Elliptic Genus and Vertex Operator Algebras
}

\author{
Chongying Dong, Kefeng Liu and Xiaonan Ma
}

\begin{abstract}
We construct bundles of modules of vertex operator algebras, and prove the rigidity and vanishing theorem for the Dirac operator on loop space twisted by such bundles. This result generalizes many previous results.
\end{abstract}

\section{INTRODUCTION}

Let $X$ be a compact smooth spin manifold. The elliptic genus of LandweberStong and Ochanine can be regarded to be the index of the formal signature operator on loop space $\mathcal{L} X$ (see [47]). It is the index of the following twisted Dirac operator on $X$

$$
D \otimes \otimes_{n \geq 1} \operatorname{Sym}_{q^{n}}\left(T X \otimes_{\mathbb{R}} \mathbb{C}\right) \otimes S(T X) \otimes \otimes_{n>0} \Lambda_{q^{n}}\left(T X \otimes_{\mathbb{R}} \mathbb{C}\right)
$$

where $S(T X)$ is the spinor bundle of $X$ and $D$ is the classical Dirac operator. Here $q$ is a parameter and for a vector bundle $E$

$$
\operatorname{Sym}_{t}(E)=1+t E+t^{2} \operatorname{Sym}^{2}(E)+\cdots, \Lambda_{t}(E)=1+t E+t^{2} \Lambda^{2}(E)+\cdots
$$

are respectively the symmetric and wedge operation of $E$. This elliptic operator should be considered as infinite number of twisted Dirac operators by taking the $q$ expansion.

The following elliptic operator

$$
D \otimes \otimes_{n \geq 1} \operatorname{Sym}_{q^{n}}\left(T X \otimes_{\mathbb{R}} \mathbb{C}\right) \otimes \otimes_{n>0} \Lambda_{ \pm q^{n-1 / 2}}\left(T X \otimes_{\mathbb{R}} \mathbb{C}\right)
$$

were also studied in [47]. It was conjectured in [47] that all these elliptic operators are rigid, generalizing the famous vanishing theorem of Atiyah-Hirzebruch

Received April 12, 2005

Dong is partially supported by NSF grants, China NSF grant 1032810 and a research grant from the Committee on Research, UC Santa Cruz.

Liu is partially supported by the Sloan Fellowship and a NSF grant. 
for the $\hat{A}$-genus. There were several rather interesting proofs of these Witten's conjectures (see [46], [8], [38], [41]). The one relevant to this paper is the proof given in [38], [39] where the main idea was to use the modular invariance of affine Kac-Moody characters.

Note that the fibers of the bundles

$$
S(T X) \otimes \otimes_{n>0} \Lambda_{q^{n}}\left(T X \otimes_{\mathbb{R}} \mathbb{C}\right) \otimes_{n>0} \Lambda_{ \pm q^{n-1 / 2}}\left(T X \otimes_{\mathbb{R}} \mathbb{C}\right)
$$

are level one highest weight integrable module for affine Kac-Moody Lie algebra $D_{l}^{(1)}$ where $l$ is half the dimension of $X$. This explains partially why the modular invariance of characters of level 1 highest weight integrable modules for affine algebra $D_{l}^{(1)}$ enters the proof of the rigidity [38].

It is well known that the level one highest weight irreducible module $L\left(\Lambda_{0}\right)$, where $\Lambda_{0}$ is the fundamental weight of $D_{l}^{(1)}$ corresponding to the index 0 , is a vertex operator algebra and the four level one highest weight integrable modules for the affine algebra $D_{l}^{(1)}$ are the irreducible modules for $L\left(\Lambda_{0}\right)$. In our case, the bundles $S(T X) \otimes \otimes_{n>0} \Lambda_{q^{n}}\left(T X \otimes_{\mathbb{R}} \mathbb{C}\right), \otimes_{n>0} \Lambda_{ \pm q^{n-1 / 2}}\left(T X \otimes_{\mathbb{R}} \mathbb{C}\right)$ are $L\left(\Lambda_{0}\right)$-bundles in the sense that each fiber is a module of $L\left(\Lambda_{0}\right)$. In this paper, we construct very general bundles such that the corresponding twisted Dirac operators is rigid. Namely, we twist the Dirac operator by a rather general class of vertex operator algebra bundles and prove the rigidity property of the corresponding elliptic operators. The main idea in the proof of rigidity theorem again is to use the modular invariance of certain trace functions in the theory of vertex operator algebras.

The study of elliptic operators on loop space twisted by a general vertex operator algebra bundle in this paper is motivated by attempting to understand the monstrous moonshine [12] geometrically. Borcherds proved [7] the ConwayNorton's moonshine conjecture for the McKay-Thompson series associated to the moonshine vertex operator algebra constructed in [26]. But there is still a lot of interest to understand the genus zero property for the McKay-Thompson series geometrically. For example, Hirzebruch proposed to realize the $J$ function as an $\hat{A}$-genus [29]. We hope that McKay-Thompson series can be realized as the Monster equivariant elliptic genus of certain elliptic operator on some orbifold [19]. This will lead to the study of elliptic operators on loop space twisted by vertex operator algebra bundles whose fibers are twisted modules for the vertex operator algebra. Another motivation for such construction is to understand the geometric meaning of elliptic cohomology by using bundles of vertex operator algebras. Some progress has been made in this direction. Actually our results indicate that elliptic cohomology should contain certain vertex operator algebra bundles. 
The setting and the argument in this paper give a uniform treatment for the Dirac operators on loop spaces when $V$ are the vertex operator algebras associated to the highest weight integrable modules for affine Kac-Moody algebras. More importantly, our results even in the case that $V$ is a lattice vertex operator algebra are totally new: many lattice vertex operator algebras are not "natural" modules for loop groups or affine Kac-Moody algebras. As far as we know, this is also the first interesting application of the algebraic theory of vertex operator algebras into geometry and topology. The ideas and results in this paper can be carried out for the orbifold elliptic genus in the setting of [19].* In this case, one uses twisted sectors or twisted modules for vertex operator algebras instead of modules. The results on modular invariance of trace functions in orbifold theory obtained in [17] is also needed.

This paper is organized as follows. In Section 1 we review the basic facts about vertex operator algebras and present some results which will be used in Section 2. In particular, we discuss the modularity of certain trace functions associated to vertex operator algebras and their representations. We also give a brief account of lattice vertex operator algebras for reader's convenience. In Section 2, we begin with a compact Lie group $G$ which acts on the vertex operator algebra as automorphisms. Using the principal $G$-bundle we construct the vertex operator algebra bundles on manifolds. We then consider the Dirac operator on the loop space twisted by the vertex operator algebra bundles and prove a rigidity theorem.

Acknowledgments. Part of this work was done while the third author was visiting UCSC. He would like to thank the Mathematics Department of UCSC for its hospitality. This project was initiated during the authors' visit to the Morningside Center of Mathematics in Beijing.

\section{Vertex operator algebras and modular inVARIANCE}

In this section we present some results about vertex operator algebras and their graded traces. We recall some details for those readers who are not very familiar with the theory of vertex operator algebras.

1.1. Vertex operator algebras and modules. We give the definitions of vertex operator algebras and their modules in this section (cf. [6], [15], [26], [48]).

${ }^{*}$ The condition $c_{1}(W)=0$ in $H^{*}(P, \mathbf{Z})$ in [19, Theorem 4.2] should be modified as following: $\operatorname{det} W$ is a trivial complex line bundle as an orbifold bundle on $X$. In fact, $\sum_{v} v \operatorname{dim} W_{v}$ is constant on each connected component of $X$ under the current condition (cf. the sentence after $[19,(4.22),(4.28)])$. 
Let $z, z_{0}, z_{1}, z_{2}$ be commuting formal variables. We shall use the basic generating function

$$
\delta(z)=\sum_{n \in \mathbb{Z}} z^{n}
$$

which is formally the expansion of the $\delta$-function at $z=1$. The fundamental (and elementary) properties of the $\delta$-function are in [26], [25] and [14].

A vertex operator algebra is a $\mathbb{Z}$-graded vector space:

$$
V=\bigoplus_{n \in \mathbb{Z}} V_{n} ; \quad \text { for } \quad v \in V_{n}, \quad n=\mathrm{wt} v
$$

such that $\operatorname{dim} V_{n}<\infty$ for all $n \in \mathbb{Z}$ and $V_{n}=0$ if $n$ is sufficiently small; equipped with a linear map

$$
\begin{aligned}
& V \rightarrow(\text { End } V)\left[\left[z, z^{-1}\right]\right] \\
& v \mapsto Y(v, z)=\sum_{n \in \mathbb{Z}} v_{n} z^{-n-1} \quad\left(v_{n} \in \text { End } V\right)
\end{aligned}
$$

and with two distinguished vectors $\mathbf{1} \in V_{0}, \omega \in V_{2}$ satisfying the following conditions for $u, v \in V$ :

$$
\begin{aligned}
& u_{n} v=0 \quad \text { for } n \text { sufficiently large; } \\
& Y(\mathbf{1}, z)=1 ; \\
& Y(v, z) \mathbf{1} \in V[[z]] \text { and } \lim _{z \rightarrow 0} Y(v, z) \mathbf{1}=v ;
\end{aligned}
$$

and there exists a nonnegative integer $l$ depending on $u, v$ such that

$$
\begin{aligned}
& \left(z_{1}-z_{2}\right)^{l}\left[Y\left(u, z_{1}\right), Y\left(v, z_{2}\right)\right]=0 \\
& {[L(m), L(n)]=(m-n) L(m+n)+\frac{1}{12}\left(m^{3}-m\right) \delta_{m+n, 0}(\operatorname{rank} V)}
\end{aligned}
$$

for $m, n \in \mathbb{Z}$, where

$$
L(n)=\omega_{n+1} \quad \text { for } \quad n \in \mathbb{Z}, \quad \text { i.e., } \quad Y(\omega, z)=\sum_{n \in \mathbb{Z}} L(n) z^{-n-2}
$$

and

$$
\begin{aligned}
& L(0) v=n v=(\operatorname{wt} v) v \quad \text { for } \quad v \in V_{n}(n \in \mathbb{Z}) \\
& \frac{d}{d z} Y(v, z)=[L(-1), Y(v, z)]=Y(L(-1) v, z) .
\end{aligned}
$$

This completes the definition. We denote the vertex operator algebra just defined by $(V, Y, \mathbf{1}, \omega)$ (or briefly, by $V$ ). The series $Y(v, z)$ are called vertex operators.

An automorphism $g$ of the vertex operator algebra $V$ is a linear automorphism of $V$ preserving $\mathbf{1}$ and $\omega$ such that the actions of $g$ and $Y(v, z)$ on $V$ are compatible in the sense that $g Y(v, z) g^{-1}=Y(g v, z)$ for $v \in V$. Then $g V_{n} \subset V_{n}$ for $n \in \mathbb{Z}$. 
The group of all automorphisms of the vertex operator algebra $V$ is denoted by $\operatorname{Aut}(V)$.

A relevant concept is derivation. A derivation of $V$ is an endomorphism $D$ : $V \longrightarrow V$ such that

$$
D\left(a_{n} b\right)=D(a)_{n} b+a_{n} D(b) \quad \text { for all } a, b \in V, n \in \mathbb{Z}
$$

and $D(\omega)=0$. In particular, $D$ preserves the gradation of $V$. So the exponential $e^{D}$ converges on $V$ and is well-defined. It is easy to see that $e^{D}$ is an automorphism.

Suppose that $V=\oplus_{n \geq 0} V_{n}$ with $V_{0}=\mathbb{C} 1$, that is, $V$ is of CFT type [18]. Then $V_{1}$ is a Lie algebra under $[u, v]=u_{0} v$ with a symmetric invariant bilinear form $(u, v)=u_{1} v$ for $u, v \in V_{1}$. Moreover, each $V_{n}$ is a $V_{1}$-module with $u$ acting as $u_{0}$. In this case $u_{0}$ is a derivation of $V$ and $e^{u_{0}}$ is an automorphism of $V$. Set

$$
N=\left\langle e^{a_{0}} \mid a \in V_{1}\right\rangle .
$$

Since $\sigma e^{a_{0}} \sigma^{-1}=e^{(\sigma a)_{0}}$ and $\operatorname{wt}(\sigma(a))=1$ for any $\sigma \in \operatorname{Aut}(V), N$ is a normal subgroup of $\operatorname{Aut}(V)$ (cf. [23]). It is conjectured in [23] that $\operatorname{Aut}(V) / N$ is a finite group. An equivalent conjecture is that the derivation algebra of $V$ is $V_{1}$.

We say that $V$ is of strong CFT type if $V$ further satisfies the condition that $L(1) V_{1}=0$. Recall from [25] that a bilinear from $(\cdot, \cdot)$ on $V$ is called invariant if

$$
(Y(u, z) v, w)=\left(u, Y\left(e^{z L(1)}\left(-z^{-2}\right)^{L(0)} v, z^{-1}\right) w\right)
$$

for $u, v, w \in V$. If $V$ is also simple, that is $V$ is an irreducible $V$-module, then there is unique nondegenerate invariant bilinear from on $V$ [35]. We shall fix a bilinear form $(\cdot, \cdot)$ on $V$ so that $(u, v)=u_{1} v$ for $u, v \in V_{1}$ (cf. [35]). It is clear from the definition that $(g u, g v)=(u, v)$ for any automorphism $g$ and $u, v \in V$.

Remark 1.1. Let $\mathfrak{g}$ be a finite dimensional simple Lie algebra and $\hat{\mathfrak{g}}=\mathfrak{g} \otimes$ $\mathbb{C}\left[t, t^{-1}\right] \oplus \mathbb{C} K$ the corresponding affine Kac-Moody Lie algebra. Let $V$ be the vertex operator algebra associated to the irreducible highest weight representation of level $m$ for $\hat{\mathfrak{g}}$. That is, $V$ is the irreducible quotient of the Verma module $U(\hat{\mathfrak{g}}) \otimes_{U(\mathfrak{g} \otimes \mathbb{C}[t]+\mathbb{C} K)} \mathbb{C}$ where $\mathbb{C}$ is the trivial module for $\mathfrak{g} \otimes \mathbb{C}[t]$-module and $K$ acts on $\mathbb{C}$ as scalar $m$. Then $V_{1}$ is isomorphic to $\mathfrak{g}$ and the bilinear form $(\cdot, \cdot)$ defined by $u_{1} v$ is the $m$ multiple of canonical bilinear form $\langle\cdot, \cdot\rangle$ (the square length of a long root in canonical bilinear form is 2 ).

Now we define admissible modules and ordinary modules for vertex operator algebras. An admissible $V$-module

$$
M=\bigoplus_{n=0}^{\infty} M(n)
$$


is a $\mathbb{Z}$-graded vector space with the top level $M(0) \neq 0$ equipped with a linear map

$$
\begin{aligned}
& V \longrightarrow(\text { End } M)\left[\left[z, z^{-1}\right]\right] \\
& v \longmapsto Y_{M}(v, z)=\sum_{n \in \mathbb{Z}} v_{n} z^{-n-1} \quad\left(v_{n} \in \text { End } M\right)
\end{aligned}
$$

which satisfies the following conditions; for $u, v \in V, w \in M, n \in \mathbb{Z}$,

$$
\begin{aligned}
& u_{n} w=0 \text { for } n \gg 0, \\
& Y_{M}(\mathbf{1}, z)=1,
\end{aligned}
$$

$$
\begin{gathered}
z_{0}^{-1} \delta\left(\frac{z_{1}-z_{2}}{z_{0}}\right) Y_{M}\left(u, z_{1}\right) Y_{M}\left(v, z_{2}\right)-z_{0}^{-1} \delta\left(\frac{z_{2}-z_{1}}{-z_{0}}\right) Y_{M}\left(v, z_{2}\right) Y_{M}\left(u, z_{1}\right) \\
=z_{2}^{-1} \delta\left(\frac{z_{1}-z_{0}}{z_{2}}\right) Y_{M}\left(Y\left(u, z_{0}\right) v, z_{2}\right)
\end{gathered}
$$

(Jacobi identity) where all binomial expressions $\left(z_{i}-z_{j}\right)^{n}$ are to be expanded in nonnegative integral powers of the second variable $z_{j}$ : This identity is interpreted algebraically as follows: if this identity is applied to a single vector of $V$ then the coefficient of each monomial in $z_{0}, z_{1}, z_{2}$ is a finite sum in $V$;

$$
u_{m} M(n) \subset M(\operatorname{wt}(u)-m-1+n)
$$

if $u$ is homogeneous. We denote the admissible $V$-module by $M=\left(M, Y_{M}\right)$.

Remark 1.2. Let $\left(M, Y_{M}\right)$ be an admissible $V$-module. Then $L(-1)$-derivation property

$$
Y_{M}(L(-1) v, z)=\frac{d}{d z} Y_{M}(v, z)
$$

holds. Moreover, the component operators of $Y_{M}(\omega, z)$ generate a copy of the Virasoro algebra of central charge rank $V$ (see [15]).

A (ordinary) $V$-module is an admissible $V$-module $M$ which carries a $\mathbb{C}$-grading induced by the spectrum of $L(0)$. That is, we have

$$
M=\bigoplus_{\lambda \in \mathbb{C}} M_{\lambda}
$$

where $M_{\lambda}=\{w \in M \mid L(0) w=\lambda w\}$. Moreover we require that $\operatorname{dim} M_{\lambda}$ is finite and for fixed $\lambda, M_{n+\lambda}=0$ for all small enough integers $n$.

A vertex operator algebra $V$ is called rational if any admissible $V$-module is a direct sum of irreducible admissible $V$-modules. It was proved in [16, Theorem 8.1] (also see [48]) that if $V$ is a rational vertex operator algebra then every irreducible admissible $V$-module is an ordinary $V$-module and $V$ has only finitely 
many irreducible admissible modules up to isomorphism. We introduce the following notions:

(1) $V$ is called holomorphic if $V$ is rational and $V$ is the only irreducible module for itself.

(2) $V$ is called $C_{2}$-cofinite if $C_{2}(V)=\left\langle u_{2} v \mid u, v \in V\right\rangle$ is of finite codimension.

It is a well known conjecture in the theory of vertex operator algebra that rationality and $C_{2}$-cofinite conditions are equivalent. A vertex operator algebra $V$ is said to be strongly rational if $V$ is of strong CFT type, rational and $C_{2^{-}}$ cofinite.

The following theorem was proved in [21].

Theorem 1.3. If $V$ is strongly rational then $V_{1}$ is a reductive algebra.

We shall fix a Cartan subalgebra $\mathfrak{h}$ of $V_{1}$.

1.2. Trace functions and modular invariance. We first review the vertex operator algebras on torus as defined in [48]. The new vertex operator algebra is denoted by $(V, Y[], \mathbf{1}, \omega-c / 24)$ where $c$ is the central charge of $V$. The new vertex operator associated to a homogeneous element $a$ is given by

$$
Y[a, z]=\sum_{n \in \mathbb{Z}} a[n] z^{-n-1}=Y\left(a, e^{z}-1\right) e^{z \mathrm{wt} a}
$$

while a Virasoro element is $\tilde{\omega}=\omega-c / 24$. Thus

$$
a[m]=\operatorname{Res}_{z}\left(Y(a, z)(\ln (1+z))^{m}(1+z)^{\mathrm{wt} a-1}\right)
$$

and

$$
a[m]=\sum_{i=m}^{\infty} c(\text { wt } a, i, m) a(i)
$$

for some scalars $c(\operatorname{wt} a, i, m)$ such that $c(\operatorname{wt} a, m, m)=1$. In particular,

$$
a[0]=\sum_{i \geq 0}\left(\begin{array}{c}
\mathrm{wt} a-1 \\
i
\end{array}\right) a(i) .
$$

We also write

$$
L[z]=Y[\omega, z]=\sum_{n \in \mathbb{Z}} L[n] z^{-n-2} .
$$


Then the $L[n]$ again generate a copy of the Virasoro algebra with the same central charge $c$. Now $V$ is graded by the $L[0]$-eigenvalues, that is

$$
V=\bigoplus_{n \in \mathbb{Z}} V_{[n]}
$$

where $V_{[n]}=\{v \in V \mid L[0] v=n v\}$. It should be pointed out that for any $n \in \mathbb{Z}$ we have

$$
\sum_{m \leq n} V_{m}=\sum_{m \leq n} V_{[m]}
$$

It is worthy to remark that if $v \in V_{n}$ is a lowest weight vector for the Virasoro algebra generated by $L(m), m \in \mathbb{Z}$ then $v \in V_{[n]}$. In particular if $L(1) V_{1}=0$ then $V_{1}=V_{[1]}$.

Let $M=\sum_{\lambda \in \mathbb{C}} M_{\lambda}$ be a $V$-module. For homogeneous $a \in V$ we define

$$
o(a)=a_{\mathrm{wt} a-1},
$$

and extend $o(a)$ to all $a$ by linearity. Let $a \in V$ we define

$$
Z_{M}(a, q)=\operatorname{Tr}_{M} o(a) q^{L(0)-c / 24}=q^{-c / 24} \sum_{\lambda \in \mathbb{C}}\left(\operatorname{Tr}_{M_{\lambda}} o(a)\right) q^{\lambda} .
$$

If $V$ is $C_{2}-$ cofinite it is proved in [48, Theorem 4.4.1] that $Z_{M}(a, q)$ converges to a holomorphic function in upper half plane with $q=e^{2 \pi i \tau}$.

Now we assume that $V$ is rational. Let $M^{1}, \ldots, M^{n}$ be the irreducible $V$ modules. Then there exist rational numbers $\lambda_{i}$ for $i=1, \ldots, n$ such that

$$
M^{i}=\sum_{p=0}^{\infty} M_{\lambda_{i}+p}^{i}
$$

(see [17, Theorem 11.1]) and $M_{\lambda_{i}}^{i} \neq 0$. For $a \in V$ we set $Z_{i}(a, q)=Z_{M^{i}}(a, q)$ for $i=1, \ldots, n$. Then

$$
Z_{i}(a, q)=q^{\lambda_{i}-c / 24} \sum_{p=0}^{\infty}\left(\operatorname{Tr}_{M_{\lambda_{i}+p}^{i}} o(a)\right) q^{p} .
$$

The following modular property is given in [48, Theorem 5.5.1].

Theorem 1.4. Assume that $V$ is rational, $C_{2}$-cofinite. Let $v \in V_{[m]}$ and $\gamma=$ $\left(\begin{array}{l}a b \\ c d\end{array}\right) \in S L(2, \mathbb{Z})$. Then $Z_{s}(v, q)$ converges to a holomorphic function in the upper half plane and there exist scalars $\gamma_{\text {st }}$ independent of $v, m$ and $\tau$ such that

$$
Z_{s}\left(v, \frac{a \tau+b}{c \tau+d}\right)=(c \tau+d)^{m} \sum_{t=1}^{n} \gamma_{s t} Z_{t}(v, \tau) .
$$


We remark that in fact the condition that $V$ is a sum of lowest weight modules for the Virasoro algebra was assumed in [48]. This condition was removed in [17].

We should also mention that Theorem 1.4 does not assert that $Z_{s}(v, q)$ is a modular form of weight $m$ on a subgroup of $S L(2, \mathbb{Z})$ of finite index. Although the modularity of $Z_{s}(v, \tau)$ is always assumed in physics, it is still an open problem in mathematics. For the discussion of the next section we introduce the following definition.

Definition 1.5. A module $M$ for vertex operator algebra $V$ is called modular if there exists a subgroup $\Gamma$ of $S L(2, \mathbb{Z})$ of finite index such that $Z_{M}(v, \gamma \tau)=$ $\psi(\gamma)(c \tau+d)^{m} Z_{M}(v, \tau)$ for $v \in V_{[m]}$ and $\gamma \in \Gamma$ where $\psi$ is a character on $\Gamma$.

The irreducible modules for well-known rational vertex operator algebras are modular (see [30] for the affine vertex operator algebras and [22] for the lattice vertex operator algebras).

Following [44] we define

$$
Z_{s}(v, u, q)=\operatorname{Tr}_{M^{s}} e^{2 \pi i(o(v)+(u, v) / 2)} q^{L(0)+o(u)+(u, u) / 2-c / 24},
$$

for $u, v \in V_{1}$. We remark that the bilinear form on $V_{1}$ used in [44] is the minus of the bilinear form used in this paper. So our $Z_{s}(v, u, q)$ has a slightly different expression although they are exactly the same as in [44]. Based on Theorem 1.4, a modular transformation law is obtained in the [44, Main theorem].

Theorem 1.6. Suppose that $V$ be a rational, $C_{2}$-cofinite vertex operator algebra of CFT type. Assume that $u, v \in V_{1}$ such that $u, v$ span an abelian Lie subalgebra of $V_{1}$. Let $\gamma=\left(\begin{array}{ll}a & b \\ c & d\end{array}\right) \in S L(2, \mathbb{Z})$. Then $Z_{s}(u, v, q)$ converges to a holomorphic function in the upper half plane and

$$
Z_{s}\left(v, u, \frac{a \tau+b}{c \tau+d}\right)=\sum_{t=1}^{n} \gamma_{s t} Z_{t}(a v+b u, c v+d u, \tau)
$$

where $\gamma_{s t}$ is the same as in Theorem 1.4.

Remark 1.7. Although the modular transformation properties of $Z_{s}(v, u, q)$ was given in [44], but the convergence of $Z_{s}(v, u, q)$ was never discussed there. We will prove in the next proposition that $Z_{s}(v, 0, q)$ is convergent in the upper half plan.

From now on we assume that $V$ is strongly rational. Recall that $\mathfrak{h}$ is a fixed Cartan subalgebra of the reductive Lie algebra $V_{1}$ (cf. Theorem 1.3). Since the homogeneous subspace of a module $M$ of $V$ is finite dimensional, $M$ is a direct sum of generalized eigenspaces for $\mathfrak{h}$. Since the restriction of the bilinear form on $V$ to $\mathfrak{h}$ is nondegenerate we can identify $\mathfrak{h}^{*}$ with $h$ via the bilinear from. 
We now define

$$
\chi_{s}(v, \tau)=\chi_{M^{s}}(v, \tau)=Z_{s}(v, 0, \tau)
$$

for $v \in V_{1}$.

Proposition 1.8. Assume that $V$ is strongly rational. Then $\chi_{s}(v, \tau)$ converges to a holomorphic function in $\mathfrak{h} \times \mathbb{H}$ where $\mathbb{H}=\{\tau \in \mathbb{C} ; \operatorname{Im} \tau>0\}$ is the upper half plane.

Proof : We first recall a result from [27, Proposition 8] on the generators of $V$. Let $\operatorname{dim} V / C_{2}(V)=m$ and $x^{1}, \ldots, x^{m}$ the coset representatives such that $L(0) x^{i}=$ $\mu_{i} x^{i}$ and $x^{i}$ is a generalized eigenvector for $\mathfrak{h}$ with eigenvalue $\alpha_{i} \in \mathfrak{h}$. Let $U^{s}$ be the space of lowest weight space of $M^{s}$. Then $M^{s}=\oplus_{p \geq 0} M_{\lambda_{s}+p}^{s}$ where $M_{\lambda_{s}}^{s}=U^{s}$. For convenience we set $u(m)=u_{\mathrm{wt} u-1-m}$ for $u \in V$ and $m \in \mathbb{Z}$. Then $M^{s}$ is spanned by

$$
x^{i_{1}}\left(m_{1}\right) \cdots x^{i_{k}}\left(m_{k}\right) U^{s}
$$

for $1 \leq i_{j} \leq m$ and $m_{1} \geq m_{2} \geq \cdots \geq m_{k}>0$ (see [37], [31], [27], [10]). Take $w \in U^{s}$ to be a generalized eigenvector for $\mathfrak{h}$ with eigenvalue $\gamma \in \mathfrak{h}$. Then the subspace $W$ spanned by

$$
x^{i_{1}}\left(m_{1}\right) \cdots x^{i_{k}}\left(m_{k}\right) w
$$

for $1 \leq i_{j} \leq m$ and $m_{1} \geq m_{2} \geq \cdots \geq m_{k}>0$ is invariant under $L(0)$ and $o(v)$ for $v \in \mathfrak{h}$. Since $U^{s}$ is finite dimensional, it is enough to prove that $\operatorname{Tr}_{W} e^{2 \pi i o(v)} q^{L(0)-c / 24}$ is holomorphic in $\mathfrak{h} \times \mathbb{H}$.

It is easy to see that

$$
\operatorname{Tr}_{W} e^{2 \pi i o(v)} q^{L(0)-c / 24} \leq q^{-c / 24+\lambda_{i}} e^{2 \pi i(v, \gamma)} \prod_{j=1}^{m} \prod_{p>0}\left(1-q^{p} e^{2 \pi i\left(v, \alpha_{j}\right)}\right)^{-1}
$$

where the inequality holds for each coefficient of the $q^{\mu} e^{2 \pi i} e^{2 \pi i\left(v, t_{1} \alpha_{1}+\cdots+t_{m} \alpha_{m}\right)}$. So it suffices to show that the power series

$$
\prod_{p>0}\left(1-q^{p} \xi\right)^{-1}
$$

is convergent absolutely for $(\xi, \tau) \in \mathbb{C}^{*} \times \mathbb{H}$. Write $\xi=e^{\alpha+i \beta}$ and $\tau=x+i y$ for $\alpha, \beta, x, y \in \mathbb{R}$ with $y>0$. Then $\left|q^{p} \xi\right|=e^{-2 \pi p y} e^{\alpha}$. It is clear now that $\prod_{p>0}\left(1-q^{p} \xi\right)^{-1}$ is absolutely convergent.

Remark 1.9. The same argument also shows that $Z_{s}(u, v, q)$ is convergent for any $u, v \in \mathfrak{h}$.

Next we discuss the transformation law for $\chi_{s}(v, \tau)$ under the modular group action. 
Proposition 1.10. Assume that $V$ is strongly rational. Then for $\gamma=\left(\begin{array}{ll}a & b \\ c & d\end{array}\right) \in$ $S L(2, \mathbb{Z}), v \in V_{1}$

$$
\chi_{s}\left(\frac{v}{c \tau+d}, \frac{a \tau+b}{c \tau+d}\right)=e^{\pi i(c(v, v) /(c \tau+d))} \sum_{j=1}^{n} \gamma_{s j} \chi_{j}(v, \tau) .
$$

In particular, if $M$ is modular and $\Gamma$ is the corresponding subgroup of $S L(2, \mathbb{Z})$, then

$$
\chi_{M}\left(\frac{v}{c \tau+d}, \frac{a \tau+b}{c \tau+d}\right)=\psi(\gamma) e^{\pi i(c(v, v) /(c \tau+d))} \chi_{M}(v, \tau)
$$

Proof : It is enough to prove that

$$
\chi_{s}\left(\frac{v}{\tau},-\frac{1}{\tau}\right)=e^{\pi i(v, v) / \tau} \sum_{j=1}^{n} S_{s, t} \chi_{t}(v, \tau),
$$

where $S_{s, t}$ corresponds to the matrix $S=\left(\begin{array}{cc}0 & -1 \\ 1 & 0\end{array}\right)$ in Theorem 1.4 and

$$
\chi_{s}(v, \tau+1)=e^{2 \pi i\left(\lambda_{s}-c / 24\right)} \chi_{s}(v, \tau) .
$$

The transformation law for $T=\left(\begin{array}{ll}1 & 1 \\ 0 & 1\end{array}\right)$ is clear. For the $S$ matrix we note from Theorem 1.6 that

$$
\chi_{s}\left(v,-\frac{1}{\tau}\right)=\sum_{t=1}^{n} S_{s, t} Z_{t}(0, v, \tau)
$$

for any $u \in \mathfrak{h}$. In particular,

$$
\chi_{s}\left(\frac{v}{\tau},-\frac{1}{\tau}\right)=\sum_{t=1}^{n} S_{s, t} Z_{t}\left(0, \frac{v}{\tau}, \tau\right)
$$

It is straightforward to verify that

$$
Z_{t}\left(0, \frac{v}{\tau}, \tau\right)=e^{\pi i(v, v) / \tau} \chi_{t}(v, \tau) .
$$

This completes the proof.

Remark 1.11. Recall Remark 1.1. In this case, the factor $e^{\pi i(v, v) / \tau}$ in Proposition 1.10 becomes to $e^{\pi i m\langle v, v\rangle / \tau}$.

Recall from Theorem 1.3 that $V_{1}$ is a reductive Lie algebra and $\mathfrak{h}$ is a Cartan subalgebra. Then $V_{1}=\mathfrak{g}_{s s} \oplus \mathfrak{g}_{a}$ is a direct sum of the semisimple ideal $\mathfrak{g}_{s s}$ and the center $\mathfrak{g}_{a}$ and $\mathfrak{h}=\mathfrak{h}_{s s} \oplus \mathfrak{g}_{a}$ where $\mathfrak{h}_{s s}$ is a Cartan subalgebra of $\mathfrak{g}_{s s}$. Since $\mathfrak{g}_{s s}$ 
acts completely on any $V$-module $M^{i}, \mathfrak{h}_{\text {ss }}$ acts on $M^{i}$ semisimply. For $\alpha \in \mathfrak{h}$ let $M^{i, \alpha}$ be the generalized eigenspace for $\mathfrak{h}$ with eigenvalue $\alpha$. Also set

$$
Q=\left\{\alpha \in \mathfrak{h} \mid V^{\alpha} \neq 0\right\} .
$$

Then $Q$ is generated by $\alpha_{1}, \ldots, \alpha_{m}$ where $\alpha_{i}$ are given in the proof of Proposition 1.8. Clearly $Q=Q_{s s} \oplus Q_{a}$ where $Q_{s s}$ is a lattice of $\mathfrak{h}_{s s}$ containing the root lattice of $\mathfrak{g}_{s s}$ and $Q_{a}$ is a lattice of $\mathfrak{g}_{a}$ consisting of eigenvalues for $\mathfrak{g}_{a}$ on $V$.

Lemma 1.12. There exist $\mu_{i} \in \mathfrak{h}$ for $i=1, \ldots, n$ such that

$$
\begin{aligned}
& V=\oplus_{\alpha \in Q} V^{\alpha}, \\
& M^{i}=\oplus_{\alpha \in Q} M^{i, \mu_{i}+\alpha} .
\end{aligned}
$$

Moreover, each $V^{\alpha}$ and $M^{i, \mu_{i}+\alpha}$ are nonzero, and $Q$ span $\mathfrak{h}$.

Proof : Since $V$ is generated by $x^{1}, \ldots, x^{m}$ we clearly have

$$
V=\oplus_{\alpha \in Q} V^{\alpha} .
$$

Showing that $V^{\alpha}$ is nonzero for any $\alpha \in Q$ is equivalent to showing that if $V^{\beta}$ and $V^{\gamma}$ are nonzero then $V^{\beta+\gamma}$ and $V^{-\beta}$ are nonzero. Observe that $u_{q} V^{\gamma} \subset V^{\beta+\gamma}$ for $u \in V^{\beta}$ and $q \in \mathbb{Z}$. It follows from [14, Proposition 11.9] that if $u$ is nonzero there exists $q \in \mathbb{Z}$ such that $u_{q} V^{\gamma}$ is nonzero. That is, $V^{\beta+\gamma}$ is nonzero.

In order to see that $V^{-\beta}$ is nonzero we notice that $V^{0} \supset V_{0}$ is nonzero. Since $V$ is simple $V$ is spanned by $u_{q} w$ for $u \in V, 0 \neq w \in V^{\beta}$ and $q \in \mathbb{Z}$ (see [20, Corollary 4.2] or [36, Proposition 4.1]). Thus $V^{-\beta}$ must be nonzero, otherwise $V^{0}$ would be zero. Since $M^{i}$ is an irreducible $V$-module the same argument can prove that $M^{i}=\oplus_{\alpha \in Q} M^{i, \mu_{i}+\alpha}$ and $M^{i, \mu_{i}+\alpha}$ is nonzero for any $\alpha \in Q$.

It remains to prove that $Q$ spans $\mathfrak{h}$. Define an invariant symmetric bilinear form $(\cdot, \cdot)_{p}$ on $V_{1}$ such that $(u, v)_{p}=\operatorname{Tr}_{V_{p}} o(u) o(v)$ for $u, v \in V$. It is proved in [21] that if $p$ is big enough the form $(\cdot, \cdot)_{p}$ is nondegenerate. Since the form is invariant, the restriction of the form $(\cdot, \cdot)_{p}$ to $\mathfrak{h}$ is also nondegenerate if $p$ is large. If $Q$ does not span $\mathfrak{h}$ then for some nonzero $u \in \mathfrak{h}, o(u)$ has only zero eigenvalue. As a result $(u, v)_{p}=0$ for all $v \in \mathfrak{h}$. This is a contradiction.

This following lemma can be found in [21].

Lemma 1.13. If $V$ is a strongly rational vertex operator algebra then each $V$ module is a completely reducible $V_{1}$-module. That is, the action of $\mathfrak{g}_{a}$ on any $V$-module is semisimple.

Let $L$ be the lattice generated by $Q$ and $\mu_{i}$ for $i=1, \ldots, n$ and $L^{\circ}=\{\alpha \in$ $\mathfrak{h} \mid(\alpha, L) \subset \mathbb{Z}\}$ the dual lattice of $L$. Then we immediately have the following consequence. 
Lemma 1.14. Let $V$ be as before. Then for any $v \in \mathfrak{h}, \alpha \in L^{\circ}$ we have

$$
\chi_{s}(v+\alpha, \tau)=\chi_{s}(v, \tau) .
$$

Here is a conjecture on $\chi_{M}(v, \tau)$.

Conjecture 1.15. If $V$ is a rational vertex operator algebra of CFT type then for any irreducible module $M$

$$
\chi_{M}(v+\alpha \tau, \tau)=e^{-2 \pi i(v, \alpha)-\pi i(\alpha, \alpha) \tau} \chi_{M}(v, \tau)
$$

for $v \in \mathfrak{h}$ and $\alpha \in L^{\circ}$.

This conjecture holds for vertex operator algebras associated to the highest weight integrable representations for affine Kac-Moody Lie algebra and for lattice vertex operator algebras. The complete reducibility of each $V$-module as a $V_{1}$ module is automatic by construction in these cases. The transformation property (1.41) for affine vertex operator algebras can be found in [30]. The transformation property (1.41) for lattice vertex operator algebras is discussed below.

Here we briefly recall the structure of lattice vertex operator algebra $V_{K}$ associated to a positive definite even lattice $K$ from [6], [26], [13]. Then $K$ is a free abelian group of finite rank with a $\mathbb{Z}$-valued positive definite symmetric bilinear form $(\cdot, \cdot)$ such that the square length of any element is even. Set $\mathfrak{h}=K \otimes_{\mathbb{Z}} \mathbb{C}$ and extend the bilinear form to $\mathfrak{h}$ by $\mathbb{C}$-linearity. Regarding $\mathfrak{h}$ as an abelian Lie algebra we consider the affine algebra $\hat{\mathfrak{h}}=\mathfrak{h} \otimes \mathbb{C}\left[t, t^{-1}\right] \oplus \mathbb{C} C$. Let $M(1)$ be the canonical irreducible $\hat{\mathfrak{h}}$-module such that $C$ acts as 1 . For $h \in \mathfrak{h}$ and $n \in \mathbb{Z}$ we set $h(n)=h \otimes t^{n}$. Then $M(1)=\mathbb{C}[h(-n) \mid h \in \mathfrak{h}, n>0]$ as vector space. Let $\mathbb{C}[K]$ be the group algebra of $K$. The lattice vertex operator algebra $V_{K}$ is defined to be $M(1) \otimes \mathbb{C}[K]$ as a vector space. We refer the reader to [6] and [26] for the definition of the vertex operators $Y(u, z)$.

We write $e^{\alpha}$ for the basis element of $\mathbb{C}[K]$ corresponding to $\alpha \in K$. For $v=$ $h_{1}\left(-n_{1}\right) \cdots h_{k}\left(-n_{k}\right) \otimes e^{\alpha}\left(h_{i} \in \mathfrak{h}, n_{i}>0, \alpha \in K\right) L(0) v=\left(\sum_{i=1}^{k} n_{i}+(\alpha, \alpha) / 2\right) v$. So $\left(V_{K}\right)_{1}$ is spanned by

$$
\left\{h(-1), e^{\alpha} \mid h \in \mathfrak{h}, \alpha \in K,(\alpha, \alpha)=2\right\} .
$$

Let $\mathfrak{h}(-1)$ be the span of $h(-1)$ for $h \in \mathfrak{h}$. Then $\mathfrak{h}(-1)$ is a Cartan subalgebra of $\left(V_{K}\right)_{1}$. We identify $\mathfrak{h}$ with $\mathfrak{h}(-1)$ in an obvious way.

It is well known that $V_{K}$ is a strongly rational vertex operator algebras (cf. [13, Theorem 2.7], [15, Theorem 3.16], [17, Proposition 12.5]). Let $K^{\circ}=\{h \in$ $\mathfrak{h} \mid(h, K) \subset \mathbb{Z}\}$ be the dual lattice of $K$. Let $K^{\circ}=\cup_{g \in K^{\circ} / K}\left(K+\beta_{g}\right)$ be the coset decomposition. For $g \in K^{\circ} / K$ we set

$$
V_{K+\beta_{g}}=M(1) \otimes \mathbb{C}\left[K+\beta_{g}\right] .
$$


Then $V_{K+\beta_{g}}$ for $g \in K^{\circ} / K$ give a complete list of irreducible modules for $V_{K}$ up to isomorphism. Now let $v=h_{1}\left(-n_{1}\right) \cdots h_{k}\left(-n_{k}\right) \otimes e^{\alpha}$ with $\alpha \in K+\beta_{g}$ we still have $L(0) v=\left(\sum_{i=1}^{k} n_{i}+(\alpha, \alpha) / 2\right) v$. Moreover, $o(h(-1))=h(0)$ acts on $v$ as $(h, \alpha)$ for $h \in \mathfrak{h}$. Note that the central charge of $V_{K}$ is the rank of $K$. Set $\chi_{g}(h, \tau)=\operatorname{Tr}_{V_{K+\beta_{g}}} e^{2 \pi i h(0)} q^{L(0)-c / 24}$. We have

$$
\chi_{g}(h, \tau)=\frac{\theta_{K+\beta_{g}}(h, \tau)}{\eta(q)^{c}}
$$

where

$$
\begin{aligned}
\theta_{K+\beta_{g}}(h, \tau) & =\sum_{\alpha \in K+\beta_{g}} e^{2 \pi i(h, \alpha)} q^{(\alpha, \alpha) / 2}, \\
\eta(q) & =q^{1 / 24} \prod_{p>0}\left(1-q^{p}\right)
\end{aligned}
$$

and $c$ is the rank of $K$. It is immediate to see that

$$
\chi_{g}(h+\alpha \tau, \tau)=e^{-2 \pi i(v, \alpha)-\pi i(\alpha, \alpha) \tau} \chi_{g}(v, \tau)
$$

for $\alpha \in K^{\circ}$.

\section{Rigidity AND VANISHING THEOREMS OF VOA ELLIPTIC GENERA}

In this section we study certain elliptic operators on loop spaces twisted by vertex operator algebra bundles. More precisely, we begin with an arbitrary strong rational vertex operator algebra $V$ and a compact Lie group $G$ which acts continuously on $V$ as automorphisms. We use the principal $G$-bundle $P$ and $V$ to form the associated sequence of vector bundles $\psi(V, P)$ on the manifold which is used to define elliptic operators. We then show that these operators are rigid under certain assumptions on the vertex operator algebra $V$. We will call the indices of these twisted elliptic operators the voa elliptic genera.

2.1. Rigidity theorem of voa elliptic genera. Let $X$ be a compact manifold and $\operatorname{dim} X=2 k$. We assume that the $S^{1}$ acts on $X$, and $T X$ has an $S^{1}$ equivariant spin structure. Let $S(T X)=S^{+}(T X) \oplus S^{-}(T X)$ be the spinor bundle of $T X$. Let $D^{X}$ be the Dirac operator on $S(T X)$. If $W$ is an $S^{1}$-equivariant complex vector bundle on $X$, we will denote by $D \otimes W$ the twisted Dirac operator of $S(T X) \otimes W($ cf. $[5, \S 3.3])$.

Recall that the elliptic operator $D \otimes W$ is called rigid if the equivariant index

$$
\operatorname{Ind}_{g}(D \otimes W)=\operatorname{Tr} g_{\mid \text {Ker } D \otimes W}-\operatorname{Tr} g_{\mid \text {Coker } D \otimes W}
$$

of $D \otimes W$ is constant with respect to $g \in S^{1}$. 
For a complex (resp. real) vector bundle $W$ on $X$, as in the introduction, we let

$$
\begin{aligned}
& \operatorname{Sym}_{t}(W)=1+t W+t^{2} \operatorname{Sym}^{2} W+\cdots, \\
& \Lambda_{t}(W)=1+t W+t^{2} \Lambda^{2} W+\cdots,
\end{aligned}
$$

be the symmetric and respectively the exterior power operations of $W$ (resp. $\left.W \otimes_{\mathbb{R}} \mathbb{C}\right)$ in $K(X)[[t]]$.

Let $V=\oplus_{n \geq 0} V_{n}$ be a strongly rational vertex operator algebra. Then the bilinear form $(a, b)=a_{1} b$ on $V_{1}$ is $\operatorname{Aut}(V)$-invariant. Recall the automorphism group $N$ of $V$ from (1.13). Let $G$ be a compact Lie group which is contained in $N$ and acts continuously on $V$ as automorphisms. Then $G$ acts on each $V$-module.

Let $P$ be an $S^{1}$-equivariant principal $G$-bundle on $X$. We define

$$
\psi(V, P)=\sum_{n \geq 0}\left(P \times_{G} V_{n}\right) q^{n} \in K(X)[[q]] .
$$

Here $P \times_{G} V_{n}$ is the associated vector bundle corresponding to the representation of $G$ on $V_{n}$. More generally, if $M^{\mu}=\oplus_{p=0}^{\infty} M_{\mu+p}^{\mu}$ is an irreducible $V$-module, we define

$$
\psi\left(M^{\mu}, P\right)=\sum_{\lambda}\left(P \times_{G} M_{\lambda}^{\mu}\right) q^{\lambda} \in K(X)\left[\left[q^{\mathbb{Q}}\right]\right] .
$$

Recall that the equivariant cohomology group $H_{S^{1}}^{*}(X, \mathbb{Q})$ of $X$ is defined by

$$
H_{S^{1}}^{*}(X, \mathbb{Q})=H^{*}\left(X \times_{S^{1}} E S^{1}, \mathbb{Q}\right),
$$

where $E S^{1}$ is the usual $S^{1}$-principal bundle over the classifying space $B S^{1}$. So $H_{S^{1}}^{*}(X, \mathbb{Q})$ is a module over $H^{*}\left(B S^{1}, \mathbb{Q}\right)$ induced by the projection $\bar{\pi}: X \times_{S^{1}}$ $E S^{1} \rightarrow B S^{1}$. Let $p_{1}(T X)_{S^{1}} \in H_{S^{1}}^{*}(X, \mathbb{Q})$ be the equivariant first Pontrjagin classes of $T X$. Also recall that

$$
H^{*}\left(B S^{1}, \mathbb{Q}\right)=\mathbb{Q}[[u]]
$$

with $u$ a generator of degree 2 . Then the $G$-invariant bilinear form ()$_{V_{1}}$ defines an $S^{1}$-equivariant characteristic class $Q\left(V_{1}\right)_{S^{1}}$ of $P$.

In the rest, we suppose that there exists $l \in \mathbb{Z}$ such that

$$
Q\left(V_{1}\right)_{S^{1}}-p_{1}(T X)_{S^{1}}=l \cdot \bar{\pi}^{*} u^{2} \quad \text { in } H_{S^{1}}^{*}(X, \mathbb{Q}) .
$$

As in [39], we call $l$ the anomaly to rigidity.

Theorem 2.1. Assume that $V$ is strong, rational vertex operator algebra and $M$ an irreducible $V$-module satisfying (1.41). If the $G$-principal bundle $P$ satisfies (2.4), then the elliptic operator

$$
D^{X} \bigotimes\left(\bigotimes_{m=1}^{\infty} \operatorname{Sym}_{q^{m}}(T X) \otimes \psi(M, P)\right)
$$


is rigid for $l \leq 0$. Moreover, its equivariant index is zero on $S^{1}$ if $l<0$, especially, its index is zero.

Remark 2.2. By combining the argument in this paper and [40, $\S 2]$, we can easily generalize Theorem 2.1 to family case, and obtain the rigidity and vanishing theorems at the equivariant Chern character level [40, Definition 2.1] for the corresponding fiberwise twisted Dirac operator of a fibration.

2.2. Proof of Theorem 2.1. For $\tau \in \mathbb{H}=\{\tau \in \mathbb{C} ; \operatorname{Im} \tau>0\}, q=e^{2 \pi i \tau}, v \in \mathbb{C}$, let

$$
\theta(v, \tau)=c(q) q^{1 / 8} 2 \sin (\pi v) \Pi_{n=1}^{\infty}\left(1-q^{n} e^{2 \pi i v}\right) \Pi_{n=1}^{\infty}\left(1-q^{n} e^{-2 \pi i v}\right)
$$

be the classical Jacobi theta functions [11], where $c(q)=\Pi_{n=1}^{\infty}\left(1-q^{n}\right)$. Set

$$
\theta^{\prime}(0, \tau)=\left.\frac{\partial \theta(v, \tau)}{\partial v}\right|_{v=0}
$$

Recall that we have the following transformation formulas of theta-functions [11]:

$$
\begin{aligned}
& \theta(t+1, \tau)=-\theta(t, \tau), \quad \theta(t+\tau, \tau)=-q^{-1 / 2} e^{-2 \pi i t} \theta(t, \tau), \\
& \theta\left(\frac{t}{\tau},-\frac{1}{\tau}\right)=\frac{1}{i} \sqrt{\frac{\tau}{i}} e^{\frac{\pi i t^{2}}{\tau}} \theta(t, \tau), \quad \theta(t, \tau+1)=e^{\frac{\pi i}{4}} \theta(t, \tau) .
\end{aligned}
$$

Let $g=e^{2 \pi i t} \in S^{1}$ be a topological generator of $S^{1}$. Let $X^{g}=\left\{X_{\alpha}\right\}$ be the fixed submanifold of the circle action. Let $i_{\alpha}: X_{\alpha} \rightarrow X$ be the natural immersion. Let $i_{\alpha}^{*}: H_{S^{1}}^{*}(X, \mathbb{Q}) \rightarrow H_{S^{1}}^{*}\left(X_{\alpha}, \mathbb{Q}\right)$ denote the induced homomorphism in equivariant cohomology. We have the following $S^{1}$-equivariant decomposition of $T X$

$$
T X_{\mid X_{\alpha}}=N_{1} \oplus \cdots \oplus N_{h} \oplus T X_{\alpha}
$$

Here $N_{\gamma}$ is a complex vector bundle such that $g$ acts on it by $e^{2 \pi i m_{\gamma} t}$. We denote the Chern roots of $N_{\gamma}$ by $2 \pi i x_{\gamma}^{j}$, and the Chern roots of $T X_{\alpha} \otimes_{\mathbb{R}} \mathbb{C}$ by $\left\{ \pm 2 \pi i y_{j}^{\prime}\right\}$. Let $\operatorname{dim}_{\mathbb{C}} N_{\gamma}=d_{\gamma}$, and $\operatorname{dim} X_{\alpha}=2 k_{\alpha}$.

Now, recall that $P$ is an $S^{1}$ equivariant $G$-principal bundle on $X$. We assume that $G$ acts on the right on $P$, and $S^{1}$ acts on the left on $P$. Let $\omega$ be an $S^{1}$-equivariant connection form on $P$, it defines a $S^{1}$-equivariant horizontal sunbundle $H P$ of $T P$. Let $\Omega=d \omega+\frac{1}{2}[\omega, \omega]$ be the curvature of $\omega$, it is a two form on $P$ with values in $\mathfrak{g}$. Let $S$ be the basis of $\operatorname{Lie}\left(S^{1}\right)=\mathbb{R}$ such that $\exp (t S)=\exp (i t)$ for $t \in \mathbb{R}$. Let $S_{X}, S_{P}$ be the vector field on $X, P$ induced by $S$. For example, $\left(S_{X} f\right)(x)=\left.\frac{d}{d \varepsilon}\right|_{\varepsilon=0} f(\exp (-\varepsilon S) x)$ for $x \in X$ and $f$ a $\mathcal{C}^{\infty}$ function on $X$.

Let $W$ be a vector space. Let $\rho: G \rightarrow \operatorname{End}(W)$ be a representation of $G$, let $\mathcal{W}=P \times_{G} W$ be the corresponding associated vector bundle. Then the connection $\omega$ induces a connection $\nabla^{\mathcal{W}}$ on $\mathcal{W}$, and the corresponding curvature is given by $\rho(\Omega)\left[5\right.$, p25]. Let $\mathcal{L}^{\mathcal{W}}(S)$ be the Lie action of $S$ on the $\mathcal{C}^{\infty}$ sections 
of $\mathcal{W}$ which is defined by $\left(\mathcal{L}^{\mathcal{W}}(S) s\right)(x)=\left.\frac{d}{d \varepsilon}\right|_{\varepsilon=0}(\exp (\varepsilon S)(s(\exp (-\varepsilon S) x)))$ for $x \in X$ and $s$ a mathcalC $C^{\infty}$ section of $\mathcal{W}$ on $X$. Then the moment of $S \in \operatorname{Lie}\left(S^{1}\right)$ is given by

$$
\mu(S)=\mathcal{L}^{\mathcal{W}}(S)-\nabla_{S_{X}}^{\mathcal{W}}
$$

Let $T P / X$ be the relative tangent bundle of the fibration $P \rightarrow X$. Let $P^{H P}$, $P^{T P / X}$ be the projections from $T P=H P \oplus T P / X$ onto $H P, T P / X$. By $[5$, p24], we know for $s \in \mathcal{C}^{\infty}(P, W)^{G}=\mathcal{C}^{\infty}(X, \mathcal{W})$. Here $\mathcal{C}^{\infty}(P, W)^{G}$ is the $G$ invariant $\mathcal{C}^{\infty}$ function on $P$ with values in $W$,

$$
\begin{aligned}
\mu(S) s=\mathcal{L}^{\mathcal{W}}(S) s-\left(P^{H P} S_{P}\right) \cdot d s=\left(S_{P}-P^{H P} S_{P}\right) \cdot d s & \\
& =\left(P^{T P / X} S_{P}\right) \cdot s=-\rho\left(\omega\left(S_{P}\right)\right) s \in \mathcal{C}^{\infty}(P, W)^{G}
\end{aligned}
$$

Now the equivariant curvature of $W$ corresponding to $S[5, \mathrm{p} 211]$ is $\rho\left(\Omega-\omega\left(S_{P}\right)\right)$. So the equivariant Chern character of $\mathcal{W}$ for $g=e^{2 \pi i t}$ is

$$
\operatorname{ch}_{g}(\mathcal{W})=\operatorname{Tr}_{W} e^{\rho\left(\frac{-1}{2 \pi i} \Omega-2 \pi t \omega\left(S_{P}\right)\right)} .
$$

Now we return to our situation. When restricted to $X_{\alpha}$ we can calculate (2.11) in the following way: when we consider $P_{\mid X_{\alpha}}$ its restriction on $X_{\alpha}$, then we can define $f: P_{\mid X_{\alpha}} \times S^{1} \rightarrow G$ by : for $(p, s) \in P_{\mid X_{\alpha}} \times S^{1}, s \cdot p=p \cdot f_{p}(s)$. Then for $p$ fixed, $f_{p}: S^{1} \rightarrow G$ is a group homeomorphism, and for $h \in G, s \in G$,

$$
h f_{p h}(s) h^{-1}=f_{p}(s) .
$$

Now, we fix $p_{0} \in P$. Then $f_{p_{0}}\left(S^{1}\right)$ is contained in a maximum torus $H$ of $G$. Let $G_{1}$ be the centralize of $f_{p_{0}}\left(S^{1}\right)$ in $G$. We choose the Cartan subalgebra $\mathfrak{h}$ of $V_{1}$ in Section 1.1 such that the Lie algebra of $H$ is contained in $\mathfrak{h}$.

By using parallel transport with respect to $H P$, from $p_{0} G_{1}$, we get an $S^{1}$ equivariant $G_{1}$-principal bundle $P_{1}$ on $X_{\alpha}$ which is a subbundle of $P_{\mid X_{\alpha}}$ and for $s \in S^{1}, f_{p}(s) \in H$ doesn't depend on $p \in P_{1}$. In fact, let $X_{t}: t \in[0,1] \rightarrow X$ be a curve such that $x_{0}$ is the projection of $p_{0}$ to $X$, and let $k_{t}$ be the lift of $x_{t}$ on $P$ along $H P$, then $k_{t}\left(p_{0} g\right)=k_{t}\left(p_{0}\right) g$ for any $g \in G$. In this way, $P_{\mid X_{\alpha}}=P_{1} \times_{G_{1}} G$ is induced by the $G_{1}$-principal bundle $P_{1}$ on $X_{\alpha}$, and recall that $H$ is the maximum torus of $G_{1}$. Let $\omega_{1}$ be the restriction of $\omega$ on $P_{1}$. Then $i \omega_{1}\left(S_{P}\right)$ is constant on $P_{1}$ which lies in the lattice $L^{\circ}$ of $\mathfrak{h}$ where the lattice $L^{\circ}$ is defined in Section 1. In the same way, the restriction of $\Omega$ on $P_{1}$ lies in $\mathfrak{g}_{1}$, the Lie algebra of $G_{1}$. So for $g=e^{2 \pi i t} \in S^{1}$,

$$
\operatorname{ch}_{g}(\mathcal{W})=\operatorname{Tr}_{W} e^{\rho\left(\frac{-1}{2 \pi i} \Omega-2 \pi \omega_{1}\left(S_{P}\right) t\right)} .
$$

We write $T=i \omega_{1}\left(S_{P}\right) \in \mathfrak{h}$. Let $U=\frac{-1}{(2 \pi i)^{2}} \Omega$. Now from (1.27) and (2.13), we know that

$$
i_{\alpha}^{*} \operatorname{ch}_{g}\left(P \times_{G} M_{\lambda}\right)=\operatorname{Tr}_{M_{\lambda}} e^{2 \pi i o(U+T t)} .
$$


Thus, the restriction of the equivariant Chern character of $\psi(M, P)$ on $X_{\alpha}$ is $(2.15) i_{\alpha}^{*} \operatorname{ch}_{g}(\psi(M, P))=\sum_{\lambda} \operatorname{Tr}_{M_{\lambda}} e^{2 \pi i o(U+T t)} q^{L(0)}=q^{c / 24} \chi_{M}(U+T t, \tau)$.

Compare with (1.34).

For $g=e^{2 \pi i t}, t \in \mathbb{R}$, and $\tau \in \mathbb{H}, q=e^{2 \pi i \tau}$, we let

6) $F_{M, P}(t, \tau)=q^{-c / 24} \operatorname{Ind}_{g}\left(D^{X} \bigotimes_{m=1}^{\infty} \operatorname{Sym}_{q^{m}}(T X-\operatorname{dim} X) \otimes \psi(M, P)\right)$.

For $f(x)$ a holomorphic function, we denote by $f\left(y^{\prime}\right)\left(T X^{g}\right)=\Pi_{j} f\left(y_{j}^{\prime}\right)$, the symmetric polynomial which gives characteristic class of $T X^{g}$, and similarly for $N_{\gamma}$. Using the Atiyah-Bott-Segal-Singer Lefschetz fixed point formula [2], (2.5), (2.14), we find for $t \in[0,1] \backslash \mathbb{Q}$

$$
\left(\mathbb{Z}_{\mathrm{A}}, \bar{T}, \boldsymbol{T}\right)(t, \tau)=(2 \pi i)^{-k} \sum_{\alpha} \int_{X_{\alpha}}\left[\theta^{\prime}(0, \tau)^{k}\left(\frac{2 \pi i y^{\prime}}{\theta\left(y^{\prime}, \tau\right)}\right)\left(T X^{g}\right) \frac{\chi_{M}(U+T t, \tau)}{\Pi_{\gamma} \theta\left(x_{\gamma}+m_{\gamma} t, \tau\right)\left(N_{\gamma}\right)}\right] .
$$

Considered as functions of $(t, \tau)$, we can obviously extend $F_{M, P}(t, \tau)$ to meromorphic functions on $\mathbb{C} \times \mathbb{H}$. and holomorphic in $\tau$. The first part of Theorem 2.1 is equivalent to the statement that $F_{M, P}(t, \tau)$ is independent of $t$. We will prove $F_{M, P}(t, \tau)$ is holomorphic on $\mathbb{C} \times \mathbb{H}$, then Theorem 2.1 will be deduced from Lemma 2.3 .

Lemma 2.3. If $Q(V)_{S^{1}}-p_{1}(T X)_{S^{1}}=l \cdot \bar{\pi}^{*} u^{2}$, then for $a, b \in 2 \mathbb{Z}$,

$$
F_{M, P}(t+a \tau+b, \tau)=e^{-\pi i l\left(a^{2} \tau+2 a t\right)} F_{M, P}(t, \tau) .
$$

Proof : By (2.7), for $a, b \in 2 \mathbb{Z}, m \in \mathbb{Z}$, we have

$$
\theta(x+m(t+a \tau+b), \tau)=e^{-\pi i\left(2 m a x+2 m^{2} a t+m^{2} a^{2} \tau\right)} \theta(x+m t, \tau) .
$$

By (2.4),

$$
(U+T t, U+T t)_{V_{1}}-\left(\sum_{j}\left(y_{j}^{\prime}\right)^{2}+\sum_{\gamma, j}\left(x_{\gamma}^{j}+m_{\gamma} t\right)^{2}\right)=l \cdot t^{2}
$$

where $(\cdot, \cdot)_{V_{1}}$ is the bilinear from on $V_{1}$.

This means

$$
\begin{aligned}
& (T, T)_{V_{1}}-\sum_{\gamma} m_{\gamma}^{2} d_{\gamma}=l, \quad(T, U)_{V_{1}}=\sum_{\gamma, j} m_{\gamma} x_{\gamma}^{j}, \\
& (U, U)_{V_{1}}=\sum_{j}\left(y_{j}^{\prime}\right)^{2}+\sum_{\gamma, j}\left(x_{\gamma}^{j}\right)^{2} .
\end{aligned}
$$

By using (1.40), (1.41), (2.17), (2.19) and (2.21), we get (2.18).

Now we will examine the modular transformation property of $F_{M, P}(t, \tau)$ under the group $S L_{2}(\mathbb{Z})$. 
For $A=\left(\begin{array}{ll}a & b \\ c & d\end{array}\right) \in S L_{2}(\mathbb{Z})$, we define its modular transformation on $\mathbb{C} \times \mathbb{H}$ by

$$
A(t, \tau)=\left(\frac{t}{c \tau+d}, \frac{a \tau+b}{c \tau+d}\right)
$$

Then the two generators $S=\left(\begin{array}{cc}0 & -1 \\ 1 & 0\end{array}\right), T=\left(\begin{array}{ll}1 & 1 \\ 0 & 1\end{array}\right)$ of $S L_{2}(\mathbb{Z})$ act on $\mathbb{C} \times \mathbb{H}$ in the following way:

$$
S(t, \tau)=\left(\frac{t}{\tau},-\frac{1}{\tau}\right), T(t, \tau)=(t, \tau+1) .
$$

Lemma 2.4. For any $A=\left(\begin{array}{ll}a & b \\ c & d\end{array}\right) \in S L_{2}(\mathbb{Z})$, we have

$$
F_{M, P}(A(t, \tau))=e^{\pi i l c t^{2} /(c \tau+d)}(c \tau+d)^{k} F_{A M, P}(t, \tau),
$$

where $A M=\sum_{\mu} a_{\mu} M^{\mu}$ is a finite complex linear combination of the irreducible $V$-modules, and we denote by

$$
\begin{gathered}
F_{A M, P}(t, \tau)=(2 \pi i)^{-k} \theta^{\prime}(0, \tau)^{k} \sum_{\mu} \sum_{\alpha} a_{\mu} \int_{X_{\alpha}}\left[\left(\frac{2 \pi i y^{\prime}}{\theta\left(y^{\prime}, \tau\right)}\right)\left(T X^{g}\right)\right. \\
\left.\frac{\chi_{M^{\mu}}(U+T t, \tau)}{\Pi_{\gamma} \theta\left(x_{\gamma}+m_{\gamma} t, \tau\right)\left(N_{\gamma}\right)}\right]
\end{gathered}
$$

the complex linear combination of the corresponding equivariant indices.

Proof : Set

$$
F(t, \tau)=\frac{\theta^{\prime}(0, \tau)}{\theta(t, \tau)}
$$

By (2.7), we get

$$
F(A(t, \tau))=(c \tau+d) e^{-c \pi i t^{2} /(c \tau+d)} F((c \tau+d) t, \tau) .
$$

By Proposition 1.10, (2.14), it is easy to see that on $X_{\alpha}$,

$$
\chi_{M}(A(U+T t, \tau))=e^{c \pi i(U+T t, U+T t)_{V_{1}} /(c \tau+d)} \chi_{A M}(U+T t, \tau) .
$$

with

$$
\chi_{A M}(U+T t, \tau)=\sum_{\mu} a_{\mu} \chi_{M^{\mu}}(U+T t, \tau)
$$


By using (2.17), (2.21), (2.26) and (2.27), we get

$$
\begin{gathered}
F_{M, P}\left(\frac{t}{c \tau+d}, \frac{a \tau+b}{c \tau+d}\right)=(2 \pi i)^{-k} \sum_{\alpha} \int_{X_{\alpha}}\left[\left(2 \pi i y^{\prime} F\left(y^{\prime}, \frac{a \tau+b}{c \tau+d}\right)\right)\left(T X^{g}\right)\right. \\
\left.\Pi_{\gamma}\left(F\left(x_{\gamma}+\frac{m_{\gamma} t}{c \tau+d}, \frac{a \tau+b}{c \tau+d}\right)\left(N_{\gamma}\right)\right) \chi_{M}\left(U+\frac{T t}{c \tau+d}, \frac{a \tau+b}{c \tau+d}\right)\right] \\
=e^{\pi i l c t^{2} /(c \tau+d)}(c \tau+d)^{k}(2 \pi i)^{-k} \sum_{\alpha} \int_{X_{\alpha}}\left[\left(2 \pi i y^{\prime} F\left((c \tau+d) y^{\prime}, \tau\right)\right)\left(T X^{g}\right)\right. \\
\left.\Pi_{\gamma}\left(F\left((c \tau+d) x_{\gamma}+m_{\gamma} t, \tau\right)\left(N_{\gamma}\right)\right) \chi_{A M}((c \tau+d) U+T t, \tau)\right]
\end{gathered}
$$

By (2.29), to prove (2.24), we only need prove the following equation,

$$
\begin{aligned}
& \int_{X_{\alpha}}\left[\left(2 \pi i y^{\prime} F\left((c \tau+d) y^{\prime}, \tau\right)\right)\left(T X^{g}\right)\right. \\
& \left.\Pi_{\gamma}\left(F\left((c \tau+d) x_{\gamma}+m_{\gamma} t, \tau\right)\left(N_{\gamma}\right)\right) \chi_{A M}((c \tau+d) U+T t, \tau)\right] \\
& =\int_{X_{\alpha}}\left[\left(2 \pi i y^{\prime} F\left(y^{\prime}, \tau\right)\right)\left(T X^{g}\right)\right. \\
& \left.\Pi_{\gamma}\left(F\left(x_{\gamma}+m_{\gamma} t, \tau\right)\left(N_{\gamma}\right)\right) \chi_{A M}(U+T t, \tau)\right] .
\end{aligned}
$$

By looking at the degree $2 k_{\alpha}$ part, that is the $k_{\alpha}$-th homogeneous terms of the polynomials in $x$ 's, $y^{\prime}$ 's and $u$ 's, on both sides, we get (2.29). The proof of Lemma 2.4 is complete.

The following lemma is a generalization of [39, Lemma 2.3],

Lemma 2.5. For any $A \in S L_{2}(\mathbb{Z})$, the function $F_{A M, P}(t, \tau)$ is holomorphic in $(t, \tau)$ for $(t, \tau) \in \mathbb{R} \times \mathbb{H}$.

Proof : Let $z=e^{2 \pi i t}$, and $N=\max _{\alpha, \gamma}\left|m_{\gamma}\right|$. Denote by $D_{N} \subset \mathbb{C}^{2}$ the domain

$$
|q|^{1 / N}<|z|<|q|^{-1 / N}, 0<|q|<1 .
$$

By (2.3), (2.17) and (2.24), we know that in $D_{N}, F_{A M, P}(t, \tau)$ has a convergent Laurent series expansion of the form

$$
\sum_{\mu} a_{\mu} q^{-c / 24+\lambda_{\mu}} \sum_{j=0}^{\infty} b_{j \mu}^{A}(z) q^{j}
$$

where $\lambda_{\mu}$ is a rational number such that $M^{\mu}=\oplus_{p=0}^{\infty} M_{\lambda_{\mu}+p}^{\mu}$ (see (1.29)) and $\left\{b_{j \mu}^{A}(z)\right\}$ are rational functions of $z$ with possible poles on the unit circle.

Now considered as a formal power series of $q$,

$$
\bigotimes_{n=1}^{\infty} \operatorname{Sym}_{q^{n}}(T X-\operatorname{dim} X) \otimes \sum_{\mu} a_{\mu} q^{-c / 24} \psi\left(M^{\mu}, P\right)=\sum_{\mu} a_{\mu} q^{-c / 24+\lambda_{\mu}} \sum_{j=0}^{\infty} V_{j, \mu}^{A} q^{j}
$$


with $V_{j, \mu}^{A} \in K_{S^{1}}(X)$. Note that the terms in the above two sums correspond to each other. Now, we apply the Atiyah-Bott-Segal-Singer Lefschetz fixed point formula to each $V_{j, \mu}^{A}$, for $t \in \mathbb{R} \backslash \mathbb{Q}$, we get

$$
b_{j \mu}^{A}(z)=\operatorname{Ind}_{z}\left(D \otimes V_{j, \mu}^{A}\right) .
$$

This implies that for $t \in \mathbb{R} \backslash \mathbb{Q}, z=e^{2 \pi i t}$,

$$
b_{j \mu}^{A}(z)=\sum_{l=-N(j)}^{N(j)} a_{l, j}^{A, \mu} z^{l} .
$$

for $N(j)$ some positive integer depending on $j$ and $a_{l, j}^{A, \mu} \in \mathbb{R}$. Since both sides are analytic functions of $z$, this equality holds for any $z \in \mathbb{C}$.

On the other hand, by multiplying $F_{A M, P}(t, \tau)$ by $f(z)=\Pi_{\alpha, \gamma}\left(1-z^{m_{\gamma}}\right)^{l^{\prime} d_{\gamma}}$ $\left(l^{\prime}=\operatorname{dim} X\right)$, we get holomorphic functions which have a convergent power series expansion of the form $\sum_{\mu} a_{\mu} q^{-c / 24+\lambda_{\mu}} \sum_{j=0}^{\infty} c_{j \mu}^{A}(z) q^{j}$, with $\left\{c_{j \mu}^{A}(z)\right\}$ polynomial functions in $D_{N}$. Comparing the above two expansions, one gets

$$
c_{j \mu}^{A}(z)=f(z) b_{j \mu}^{A}(z)
$$

for each $j$. So by the Weierstrass preparation theorem, we get $F_{A M, P}(t, \tau)$ is holomorphic in $D_{N}$.

Lemma 2.6. $F_{M, P}(t, \tau)$ is holomorphic on $\mathbb{C} \times \mathbb{H}$.

Proof : Recall that by Proposition 1.8, $\chi_{M}(v, t)$ is holomorphic on $\mathfrak{h} \times \mathbb{H}$. From their expressions, we know the possible polar divisors of $F_{M, P}$ in $\mathbb{C} \times \mathbb{H}$ are of the form $t=\frac{m}{n}(c \tau+d)$ with $m, n, c, d$ integers and $(c, d)=1$ or $c=1$ and $d=0$.

We can always find integers $a, b$ such that $a d-b c=1$, and consider the matrix $A=\left(\begin{array}{cc}d & -b \\ -c & a\end{array}\right) \in S L_{2}(\mathbb{Z})$. By $(2.23)$,

$$
F_{A M, P}(t, \tau)=(-c \tau+a)^{-k} F_{M, P}\left(\frac{t}{-c \tau+a}, \frac{d \tau-b}{-c \tau+a}\right) .
$$

Now, if $t=\frac{m}{n}(c \tau+d)$ is a polar divisor of $F_{M, P}(t, \tau)$, then one polar divisor of $F_{A M, P}(t, \tau)$ is given by

$$
\frac{t}{-c \tau+a}=\frac{m}{n}\left(c \frac{d \tau-b}{-c \tau+a}+d\right),
$$

which exactly gives $t=m / n$. This contradicts Lemma 2.5, and completes the proof of Lemma 2.6.

Proof of Theorem 2.1: By Lemma 2.6, $F_{M, P}$ is holomorphic on $\mathbb{C} \times \mathbb{H}$. For fixed $\tau \in \mathbb{H}$, if $F_{M, P}(\cdot, \tau)$ isn't identically zero, we let $\delta$ be the contour 
$z_{0}+2 s, z_{0}+2+2 s \tau, z_{0}+2+2(1-s) \tau, z_{0}+2(1-s) \tau(s \in[0,1])$, such that $F_{M, P}(\cdot, \tau)$ does not have any zero on $\delta$. Then by $(2.18)$,

$$
\frac{1}{2 \pi i} \int_{\delta} \frac{1}{F_{M, P}(t, \tau)} \frac{\partial}{\partial t} F_{M, P}(t, \tau) d t=4 l .
$$

This means that $F_{M, P}(t, \tau)$ has exactly $4 l$ zeros inside $\delta$. Therefore, if $l<0, F_{M, P}$ must be identically zero. If $l=0, F_{M, P}(t, \tau)$ is a double periodic holomorphic function, it must be independent of $t$. Thus we get Theorem 2.1.

The reader may have noticed that we do not assume in Theorem 2.1 the modularity of $\chi_{M}(v, \tau)$. When $V$ is the vertex operator algebra associated to the highest weight integrable representation for the affine Kac-Moody algebra $D_{l}^{(1)}$ it was also proved in [39, Theorem 5] that $F_{M}(v, \tau)$ is a holomorphic Jacobi form by using the fact that $\chi_{M}(v, \tau)$ is modular. It turns out the same result holds in our setting under the assumption that $M$ is modular. See Definition 1.5.

Recall that a (meromorphic) Jacobi form of index $m$ and weight $l$ over $L \rtimes \Gamma$, where $L$ is an integral lattice in the complex plane $\mathbb{C}$ preserved by the modular subgroup $\Gamma \subset S L_{2}(\mathbb{Z})$, is a (meromorphic) function $F(t, \tau)$ on $\mathbb{C} \times \mathbb{H}$ such that

$$
\begin{aligned}
& F\left(\frac{t}{c \tau+d}, \frac{a \tau+b}{c \tau+d}\right)=\psi(A)(c \tau+d)^{l} e^{2 \pi i m\left(c t^{2} /(c \tau+d)\right)} F(t, \tau), \\
& F(t+\lambda \tau+\mu, \tau)=e^{-2 \pi i m\left(\lambda^{2} \tau+2 \lambda t\right)} F(t, \tau)
\end{aligned}
$$

where $(\lambda, \mu) \in L, A=\left(\begin{array}{ll}a & b \\ c & d\end{array}\right) \in \Gamma$, and $\psi(A)$ is a character of $\Gamma$. If $F$ is holomorphic on $\mathbb{C} \times \mathbb{H}$, we say that $F$ is a holomorphic Jacobi form.

Theorem 2.7. Let $X, V, M$ and $P$ be as in Theorem 2.1. Assume that $M$ is modular, then $F_{M, P}$ is a holomorphic Jacobi form of index $l / 2$ and weight $k$ over $(2 \mathbb{Z})^{2} \rtimes \Gamma$, here $\Gamma$ is the subgroup of $S L(2, \mathbb{Z})$ such that $\chi_{M}(v, \tau)$ is modular over $\Gamma$.

Proof : Recall that $\Gamma$ is the subgroup of $S L(2, \mathbb{Z})$ which defines the modular vertex operator algebra $V$. Then for any $A=\left(\begin{array}{ll}a & b \\ c & d\end{array}\right) \in \Gamma$, we have

$$
\chi_{M}(A(U+T t, \tau))=\psi(A) e^{c \pi i(U+T t, U+T t)_{V_{1}} /(c \tau+d)} \chi_{M}(U+T t, \tau) .
$$

Now, by (2.26), (2.40), as Lemma 2.4, we get

$$
F_{M, P}(A(t, \tau))=\psi(A)(c \tau+d)^{k} e^{\pi i l c t^{2} /(c \tau+d)} F_{M, P}(t, \tau) .
$$

By Lemmas 2.3, 2.6, (2.41), we get Theorem 2.7. 


\section{REFERENCES}

[1] Atiyah M.F., Hirzebruch F., Spin manifolds and groups actions, Essays on topology and Related Topics, Memoirs dédié à Georges de Rham (ed. A. Haefliger and R. Narasimhan), Springer-Verlag, New York-Berlin (1970), 18-28.

[2] Atiyah M.F., Singer I.M., The index of elliptic operators IV. Ann. Math. 93 (1971), 119-138.

[3] Ando M., Basterra M., The Witten genus and equivariant elliptic cohomology. Math. Z. 240 (2002), 787-822.

[4] Ando M., Hopkins M. J., and N. P. Strickland N. P., Elliptic spectra, the Witten genus, and the theorem of the cube, Invent. math. 146 (2001), 595-687.

[5] Berline N., Getzler E. and Vergne M., Heat kernels and the Dirac operator, Grundl. Math. Wiss. 298, Springer, Berlin-Heidelberg-New York 1992.

[6] Borcherds R., Vertex algebras, Kac-Moody algebras, and the Monster, Proc. Natl. Acad. Sci. USA 83 (1986), 3068-3071.

[7] Borcherds R., Monstrous moonshine and monstrous Lie superalgebras, Invent. Math. 109 (1992), 405-444.

[8] Bott R. and Taubes C., On the rigidity theorems of Witten, J. AMS. 2 (1989), 137-186.

[9] Brylinski, Representations of loop groups, Dirac operators on loop spaces and modular forms, Topology 29 (1990), 461-480.

[10] Buhl G., A generating set of modules for vertex operator algebras, J. Algebra 254 (2002), $125-151$.

[11] Chandrasekharan K., Elliptic functions, Springer, Berlin, 1985.

[12] Conway J. H. and Norton S. P., Monstrous Moonshine, Bull. London. Math. Soc. 12 (1979), 308-339.

[13] Dong C., Vertex algebras associated with even lattices, J. Algebra 161 (1993), 245-265.

[14] Dong C. and Lepowsky J., Generalized Vertex Algebras and Relative Vertex Operators, Progress in Math. Vol. 112, Birkhäuser, Boston 1993.

[15] Dong C., Li H. and Mason G., Regularity of rational vertex operator algebras, Adv. in Math. 132 (1997), 148-166.

[16] Dong C., Li H. and Mason G., Twisted representations of vertex operator algebras, Math. Ann. 310 (1998), 571-600.

[17] Dong C., Li H. and Mason G., Modular invariance of trace functions in orbifold theory and generalized moonshine, Comm. Math. Phys. 214 (2000), 1-56.

[18] Dong C., Li H., Mason G. and Montague P., The radical of a vertex operator algebra, in: Proc. of the Conference on the Monster and Lie algebras at The Ohio State University, May 1996, ed. by J. Ferrar and K. Harada, Walter de Gruyter, Berlin- New York, 1998, 17-25. York, 1998.

[19] Dong C., Liu K. and Ma X., On orbifold elliptic genus, Contemp. Math. 310 (2002), 87-106.

[20] Dong C. and Mason G., On quantum Galois theory, Duke Math. J. 86 (1997), 305-321.

[21] Dong C. and Mason G., Rational vertex operator algebras and effective central charge, IMRN 56 (2004), 2989-3008.

[22] Dong C., Mason G. and Nagatomo K., Quasi-modular forms and trace functions associated to free Boson and lattice vertex operator algebras, IMRN 8 (2001), 409-427.

[23] Dong C. and Nagatomo K., Automorphism groups and twisted modules for lattice vertex operator algebra, Contemp. Math. 268 (1999), 117-133.

[24] Eichler M., and Zagier D., The theory of Jacobi forms, Birkhauser, Basel, 1985.

[25] Frenkel I.B., Huang Y. and Lepowsky J., On axiomatic approach to vertex operator algebras and modules, Mem. Amer. Math. Soc. 104, 1993.

[26] Frenkel I.B., Lepowsky J. and Meurman A., Vertex operator algebras and the Monster, Academic Press, 1988. 
[27] Gaberdiel M. and Neitzke A., Rationality, quasirationality and finite W-algebras, Comm. Math. Phys. 238 (2003), 305-331.

[28] Gong D., Liu K., Rigidity of higher elliptic genera, Annals of Global Analysis and Geometry 14 (1996), 219-236.

[29] Hirzebruch F., Berger T., Jung R., Manifolds and Modular Forms. Vieweg 1991.

[30] Kac V., Infinite-dimensional Lie algebras, Cambridge Univ. Press, London, 1991.

[31] Karel M. and Li H., Certain generating subspaces for vertex operator algebras, J. Algebra 217 (1999), 393-421.

[32] Landweber P.S., Elliptic Curves and Modular forms in Algebraic Topology, Landweber P.S., SLNM 1326, Springer, Berlin.

[33] Landweber P.S., Elliptic cohomology and modular forms, in Elliptic Curves and Modular forms in Algebraic Topology, Landweber P.S., SLNM 1326, Springer, Berlin, 107-122.

[34] Lawson H.B., Michelsohn M.L., Spin geometry, Princeton Univ. Press, Princeton, 1989.

[35] Li H., Symmetric invariant bilinear forms on vertex operator algebras, J. Pure Appl. Algebra 96 (1994), 279-297.

[36] Li H., An approach to tensor product theory for representations of a vertex operator algebra, Ph.D. thesis, Rutgers University, 1994.

[37] Li H., Some finiteness properties of regular vertex operator algebras. J. Algebra 212 (1999), 495-514.

[38] Liu K., On elliptic genera and theta-functions, Topology. 35 (1996), 617-640.

[39] Liu K., On Modular invariance and rigidity theorems, J. Diff. Geom. 41 (1995), 343-396.

[40] Liu K., Ma X., On family rigidity theorems I. Duke Math. J. 102 (2000), 451-474.

[41] Liu K., Ma X. and Zhang W., Rigidity and Vanishing Theorems in K-Theory, C. R. Acad. Sci. Paris, Série I 330 (2000), 301-305.

[42] Liu K., Ma X. and Zhang W., Rigidity and Vanishing Theorems in K-Theory, Comm. Anal. Geom., 11 (2003), 121-180.

[43] Liu K., Ma X. and Zhang W., Spin ${ }^{c}$ Manifolds and Rigidity Theorems in K-Theory, Asian J. Math. 4 (2000), 933-960.

[44] Miyamoto, M., A modular invariance on the theta functions defined on vertex operator algebras, Duke Math. J. 101 (2000), 221-236.

[45] Ochanine S., Genres elliptiques equivariants, in Elliptic Curves and Modular forms in Algebraic Topology, Landweber P.S., SLNM 1326, Springer, Berlin, 107-122.

[46] Taubes C., $S^{1}$-actions and elliptic genera, Comm. Math. Phys. 122 (1989), 455-526.

[47] Witten E., The index of the Dirac operator in loop space, in Elliptic Curves and Modular forms in Algebraic Topology, Landweber P.S., SLNM 1326, Springer, Berlin, 161-186.

[48] Zhu Y., Modular invariance of characters of vertex operator algebras, J. AMS 9, (1996), 237-301.

Chongying Dong

Mathematics Department, University of California, Santa Cruz, CA 95064, U.S.A. E-mail: dong@math.ucsc.edu

Kefeng Liu

Department of Mathematics, UCLA,CA 90095-1555, USA

E-mail: liu@math.ucla.edu 
Xiaonan Ma

Centre de Mathématiques de Laurent Schwartz, UMR 7640 du CNRS, Ecole Polytechnique, 91128 Palaiseau Cedex, France

E-mail: ma@math.polytechnique.fr 